(CDU, FDP und Grüne) und einen festen Betrag an die Kreis-, Landes- und Bundespartei abgibt, oder der Beitrag wie in der SPD zentral von der Bundespartei eingezogen und nach festen Sätzen und besonderem Bedarf an die unteren Gliederungen abgegeben wird. ${ }^{1}$ Bei zentralem Einzug und Einnahmen aus eigenen Betrieben werden keine „schwarzen Kassen“ gebraucht. Ob das System der CDU bis $2000^{2}$ auch auf die Beziehungen zu den ostdeutschen Landesverbänden angewandt wurde, wird nicht einmal geprüft. Dies läge nahe, denn mit dem Verzicht des Bundesvorstands auf das Vermögen der Blockpartei gerieten die ostdeutschen Landesverbände an den Tropf der Bundespartei. Auch Information über den Umgang mit dem Vermögen - Immobilien, Büros und Druckereien - der Altparteien in FDP und PDS wären wichtig gewesen. So kämen zu den Differenzierungen zwischen Groß- und- Kleinparteien, Regierungs- und Oppositionsparteien noch die zwischen den Altparteien und Neugründungen und deren Umgang mit der Vergangenheit.

Im Fazit kann Träger uneingeschränkt zugestimmt werden. Die Beziehung der Landesverbände zu ihren Bundesparteien bleibt eine spannende große Forschungsaufgabe. $\mathrm{Zu}$ wünschen wäre allerdings ein parteien- und landesspezifischer Zugang, der über statistische Daten hinaus „den Kampf um Karrieren und Interessen, Verfügung über Geld, Personal, Ämter, Sachmittel, Informations- und Kommunikationskanäle“ (S. 20) spiegelt. Dazu müsste auch auf die Bedeutung der Bundestagsabgeordneten eingegangen werden, die mit dem Amt, den Wahlkreisbüros und ihren finanziellen Beiträgen eine nicht zu vernachlässigende Größe für den Einfluss eines Landesverbandes sind. Zu klären wäre des Weiteren, worin die spezifisch ostdeutsche Interessenvertretung besteht, die auch über die nicht einbezogenen Ostberliner Vertreter erfolgte. Trägers Dissertation zeigt, wie viele Faktoren in die innerparteiliche Willensbildung eingehen. Sie regt durch ihre Materialfülle in viele Richtungen an und demonstriert, wie dringend ein theoretisches Konzept innerparteilicher Demokratie gebraucht wird.

Ingrid Reichart-Dreyer

1 Vgl. „Entscheidungen des Parlaments in eigener Sache“, eine Tagung der Deutschen Vereinigung für Parlamentsfragen und der Deutschen Gesellschaft für Gesetzgebung, Diskussion der Beiträge von Hans Hugo Klein, Josef Isensee, Siegfried Broß und Wolfgang Rudzio, in: ZParl, 31. Jg. (2000), H. 2, S. $441-466$, S. 461.

2 Vgl. Frank Bösch, Macht und Machtverlust in der CDU, Stuttgart 2002, S. 185 ff.

\title{
Parteien und kommunaler Parlamentarismus: spannende Stadtgeschichte Frankfurts
}

\author{
Tüffers, Bettina: Von der Römerkoalition zur Parteienkonkurrenz. Geschichte der Frankfurter \\ Stadtverordnetenversammlung, Band IV, 1946-1989 (Band 24 der Schriften der Frankfurter \\ Historischen Kommission), Verlag Waldemar Kramer, Frankfurt am Main 2011, 294 Seiten, \\ $€ 28$,-
}

Gemeindevertretungen nur als Organe der Selbstverwaltung zu betrachten, greift für Bettina Tüffers zu kurz, wie sie mit ihrem zeithistorischen Beitrag zur Geschichte der Kommunalpolitik am Beispiel Frankfurts zwischen 1946 und 1989 zeigt. Rückt man die Rolle der 
Parteien in den Fokus der Analyse, so entsteht eine Perspektive auf die Stadtverordnetenversammlung, die ihren Charakter als Kommunalparlament verdeutlicht, auch wenn ihnen die Legislativfunktion fehlt. Tüffers schildert die Geschichte des Frankfurter Stadtparlaments entlang seiner zunehmenden Politisierung und der wachsenden Dominanz der Parteipolitik. Empirisch stützt sich die Arbeit auf fast 600 Wortprotokolle der Stadtverordnetenversammlung sowie auf die Auswertung der lokalen Presseberichterstattung. Illustriert wird die Argumentation mit treffenden Bildern und Karikaturen.

Anstatt einer langatmigen Stadtgeschichte werden zentrale politische Debatten rekonstruiert, die die Entwicklung Frankfurts geprägt haben. Hierzu gehören in erster Linie zahlreiche Bauprojekte wie der Wiederaufbau der Stadt, der Wohnungsbau und in diesem Zuge der Bau der Nordweststadt, die U-Bahn oder die Altstadt- und Römerbergbebauung sowie der Wiederaufbau der Alten Oper. Besonders pointiert werden in diesem Zusammenhang die städtebaulichen Differenzen zwischen SPD und CDU herausgearbeitet. Während sich die CDU für innerstädtische Prestigeprojekte und zahlreiche historische Rekonstruktionen stark machte, präferierte die SPD den Wohnungsbau und die Errichtung moderner Gebäude in der Innenstadt. Aber auch Themen wie Bürgerinitiativen, Studentenproteste, der Bau der Startbahn West und andere gesellschaftspolitische Auseinandersetzungen werden aufgegriffen, um die Entwicklung der Stadt zu dokumentieren. Darüber hinaus geht Tüffers auf die Personalrekrutierungsfunktion des Kommunalparlamentes ein. Sie zeichnet Karrierewege nach, die dort ihren Anfang nahmen und sich im Frankfurter Magistrat oder auch im Land- und Bundestag fortsetzten. Über mehr als vierzig Jahre hinweg wird zudem deutlich, wie die „Politisierung des Gemeinwesen“" (S. 12) immer wieder die Hessische Gemeindeordnung (HGO) beeinflusste. Diese wirkte streckenweise nicht normativ, sondern passte sich nachholend der Realität in Frankfurt an, beispielsweise wenn es um die politische Praxis der Fraktionsbildung ging.

Das Jahr vor der ersten Kommunalwahl 1946 beschreibt Tüffers als „Stunde null mit bekannten Gesichtern“: Die amerikanischen Alliierten, die ihr Hauptquartier in der Mainmetropole errichtet hatten, installierten schnell einen Bürgerrat ein, der sich überwiegend aus Kommunalpolitikern zusammensetzte, die bereits vor 1933 in Frankfurt politisch aktiv gewesen waren. Der weitere Verlauf der Geschichte des Kommunalparlaments bis 1989 lässt sich in zwei Phasen einteilen: Bis 1972 trug die politische Auseinandersetzung vor allem Züge der Konkordanzdemokratie, dann schlug sie in Konfliktaustragung um und lässt sich seitdem als Parteienkonkurrenz charakterisieren.

Die Phase von 1946 bis 1972 wurde von der „Römerkoalition“ geprägt und zeichnete sich dadurch aus, dass der hauptamtliche Magistrat durchgehend von Mitgliedern von SPD, CDU und FDP besetzt wurde, ohne dass es Koalitionsabsprachen oder einen Koalitionsvertrag gegeben hätte. Die Folge war ein ,wenig streitträchtiges Miteinander in der Stadtverordnetenversammlung“ (S. 21), was aber gleichzeitig zu der „schizophrenen Situation, dass die Parteien einander regelmäßig heftig kritisierten, anstehende Projekte später aber dennoch einstimmig oder zumindest einvernehmlich verabschiedeten" (S. 252), führte. Der durch Konsens unterbundene Parteienwettbewerb erwies sich als problematisch, da vielfach die Suche nach politischen Handlungsalternativen ausblieb. Dies rief Unzufriedenheit bei den Bürgern hervor und bewirkte schließlich die Gründung der Bürgerinitiative „Aktionsgemeinschaft Westend“, die überregional Bekanntheit erlangte.

Bereits zu Beginn der 1970er Jahre kündigte sich das Ende der „Römerkoalition“ an. Die politischen Veränderungen gingen allerdings mehr von den beiden Volksparteien selbst 
aus als von ihren Fraktionen im Stadtparlament. „Mit dem Generationenwechsel und unter dem Eindruck der allgemeinen 68er-Proteststimmung im Land kam in den Frankfurter Parteien eine Entwicklung in Gang, die das politische Klima in der Stadt grundlegend verändern und 1972 in der Beendigung der jahrzehntelangen Zusammenarbeit von SPD und CDU gipfeln sollte“ (S. 151). Schließlich verweigerte der Parteitag der SPD die Zustimmung zu einer weiteren Zusammenarbeit mit den Christdemokraten und lehnte die Wahl zweier CDU-Dezernenten ab. Das Ende der Koalition wurde vor allem von der Jungen Union innerhalb der CDU an der Spitze als Befreiung empfunden. Die parteipolitische Polarisierung wurde dadurch verschärft, dass es 1977 erstmals zu einem Wechsel in Frankfurt kam und die CDU den Oberbürgermeister stellte. Dennoch band die CDU weiterhin SPD-Dezernenten, wie beispielsweise den Kulturpolitiker Hilmar Hoffmann, in den Magistrat ein. Auch das Parteiensystem geriet in Bewegung, als 1981 die Grünen erstmals in den Römer einzogen und die FPD sechzehn Jahre lang pausieren musste. Tüffers zeichnet den Weg der Parlamentarisierung der Grünen in Frankfurt mit dem Sieg der Realos über die Fundis nach und zeigt in Anlehnung an Joachim Raschke, wie Parlamente als „Konventionsmaschine" wirken (S. 254).

Nachdem Tüffers den dritten Band in der Reihe der Geschichtsschreibung des Frankfurter Stadtparlamentes seit 1867 vorgelegt hat (herausgegeben von der Frankfurter Historischen Kommission), freut man sich schon auf die Fortsetzung bis in die Gegenwart. Einzig störend an der im Grunde klugen und detailreichen Darstellungsweise sind manche übertriebenen eigenen Wertungen der Autorin, beispielsweise wenn sie die durch den Einzug der Grünen ins Parlament veränderte Debattenkultur als „überhand nehmende Monstersitzungen" und als fatale Mischung beschreibt (S. 213). Auch ihre Interpretation, bei der Lektüre eines Protokolls „das kollektive Aufstöhnen der anderen zu hören“ (S. 227), gehört zu den störenden Seitenhieben, die man bei einer Überarbeitung hätte streichen sollen.

Arijana Neumann

\section{Parteien und Parteiensysteme: vielleicht doch das perfekte Lehrbuch}

Detterbeck, Klaus: Parteien und Parteiensystem, UVK Verlagsgesellschaft mbH, Konstanz 2011, 272 Seiten, €19,90.

Der Autor wollte eine Einführung in die Parteienforschung schreiben. Vor diesem Hintergrund muss die überschaubare (wenngleich überlange) Gliederung seines Werkes gelobt werden, in die tatsächlich die wichtigsten Felder dieser Forschungsrichtung eingeflossen sind: die Parteienbegriffe, die Binnenorganisation, die Ursprünge, Funktionen, Entwicklungsetappen und Ideologien der Parteien, der elektorale Prozess und die Parteiensysteme in verschiedenen institutionellen Systemzusammenhängen. Klaus Detterbeck veranschaulicht zudem die Hauptaussagen der klassischen Parteienliteratur sowie statistische Daten mittels eigens erstellter Abbildungen und Tabellen - in der Absicht, die auf visuelle Reize stark reagierende Generation der Bachelor- und Masterstudiengänge zu erreichen. Nach dem Vorbild vieler US-amerikanischer Lehrbücher fügt er zudem jedem Kapitel einen Fragenkatalog und auch Links zum Erlernen und Vertiefen des Gelesenen hinzu. 